\title{
Windowed phase unwrapping using a first-order dynamic system following iso-phase contours
}

\author{
Julio C. Estrada, ${ }^{1, \star}$ Javier Vargas, ${ }^{2}$ J. Mauricio Flores-Moreno, ${ }^{1}$ and J. Antonio Quiroga ${ }^{3}$ \\ ${ }^{1}$ Centro de Investigaciones en Optica A. C., Loma del bosque 115 Col. Lomas del campestre, \\ Leon Guanajuato 371150, Mexico \\ ${ }^{2}$ Biocomputing Unit, Centro Nacional de Biotecnologia-CSIC, C/ Darwin 3, Cantoblanco (Madrid) 28049, Spain \\ ${ }^{3}$ Optics Department, Universidad Complutense de Madrid, 28040, Spain \\ ${ }^{*}$ Corresponding author: julio@ cio.mx
}

Received 7 August 2012; accepted 18 September 2012;

posted 26 September 2012 (Doc. ID 173965); published 24 October 2012

\begin{abstract}
In this work, we show a windowed phase-unwrapping technique that uses a first-order dynamic system and scans the phase following its iso-phase contours. In previous works, we have shown that low-pass first-order dynamic systems are very robust and useful in phase-unwrapping problems. However, it is well known that all phase-unwrapping methods have a minimum signal-to-noise ratio that they tolerate. This paper shows that scanning the phase within local windows and using a path following strategy, the first-order unwrapping method increases its tolerance to noise. In this way, using the improved approach, we can unwrap phase maps where the basic dynamic phase-unwrapping system fails. Tests and results are given, as well as the source code in order to show the performance of the proposed method. (C) 2012 Optical Society of America
\end{abstract}

OCIS codes: $\quad 100.5088,120.5050$.

\section{Introduction}

Modulating information in two-dimensional (2D) sinusoidal signals is very common in areas such as optical interferometry [1]. Digital demodulation techniques are used to recover the phase information. However, the most-used demodulation techniques recover the phase modulus $2 \pi$, or in other words, wrapped into the range $(-\pi, \pi)[2, \underline{3}]$. A phase-unwrapping process is needed to recover the phase information in its full dynamic range. The phase-unwrapping process is easily explained in $1 \mathrm{D}$ as a line integration of the wrapped phase differences $[4,5]$. However, in practice the $2 \mathrm{D}$ phaseunwrapping process is not an easy task. One of the main obstacles for unwrapping the phase correctly is the noise that corrupts the wrapped phase.

$1559-128 \mathrm{X} / 12 / 317549-05 \$ 15.00 / 0$

(C) 2012 Optical Society of America
The phase unwrapping is so important that if it can not be carried out correctly, optical tests are discarded and optical setups are redesigned, if possible. One of the first ideas for unwrapping 2D noisy wrapped phases consists in locating the sites that have branch cuts [6-8]. The branch cuts are determined by the residues (or discontinuities) present in the wrapped phase. A site is labeled as a residue if it has a phase discontinuity or inconsistency around it and the branch cut is the path between positive a negative residues [8]. After locating the branch cuts, the unwrapping method scans the phase without crossing these branch cuts, tolerating in this way certain levels of noise. On the other hand, as the phase-unwrapping process is an integration of the wrapped phase differences, global integration techniques, modeled by least-squares functionals, were proposed [9-11]. The main drawback of these techniques is that they typically underestimate the dynamic range of the unwrapped phase. More recently, we have shown some works that present the theory 
and implementation of 2D low-pass dynamic systems for phase unwrapping [12-14]. There, we use a basic infinite impulse response low-pass filter adapted for unwrapping the phase in $2 \mathrm{D}$. These techniques present a noise-tolerant phase-unwrapping system that filters noise and unwraps the phase in the same process. However, there is not a perfect phaseunwrapping system and all systems have a minimum signal-to-noise ratio in which they start failing. In this paper, we improve our first-order dynamic system making it more robust to noise. The improved algorithm scans the wrapped phase within windows, and uses a path-following strategy that consists in following the iso-phase contours. Actually, phaseunwrapping methods that use a path-following strategy have been used before [15]. Here, we are going to use the gradient magnitude of the wrapped phase to follow the iso-phase contours. Processing the phase in this way, the herein presented technique obtains the expected results on those cases where the basic first-order dynamic system starts failing. In the next section, we will provide the basic ideas about the windowed phase-unwrapping method using our first-order dynamic system, and the method for following the iso-phase contours. Afterward, we show some tests and preliminary results to demonstrate the performance of the proposed method. Finally, conclusions and commentaries are drawn. A link to download the $\mathrm{C}++$ source code is given in [16].

\section{Method}

\section{A. 2D First-Order Dynamic System}

The 2D first-order dynamic system used for phase unwrapping can be written as follows:

$$
\begin{aligned}
\hat{\phi}(x, y)= & \sum_{m=x-1}^{x+1} \sum_{n=y-1}^{y+1}\{\hat{\phi}(m, n) \\
& -\tau W[\phi(x, y)-\hat{\phi}(m, n)]\} s(m, n),
\end{aligned}
$$

where $\hat{\phi}(x, y)$ is the obtained unwrapped phase at site $(x, y), x$ and $y$ are integers, $\phi(x, y)$ is the wrapped phase that is being processed at site $(x, y), \tau$ is the parameter that controls the bandwidth of the system for $|\tau|<1$, and $s(x, y)$ is a flag function that is 1 if the site $(x, y)$ has already been unwrapped, and 0 otherwise $[12,13]$. This first-order dynamic system for phase unwrapping was published first in [12]. A comparison with other sequential phase-unwrapping methods can be found in [14] and a variant of this recursive system and its analysis of the frequency response can be found in [13].

As stated in the previous works, to obtain the unwrapped phase using the dynamic system of Eq. (1), we scan the wrapped phase map visiting sequentially each site $(x, y)$ using a simple row-by-row strategy. Since for the initial point we do not have any previously processed site, it is taken directly the phase value that has the wrapped phase map in that point. As actually the dynamic system shown in Eq. (1) is a low-pass filter, it can deal with phase inconsistencies of the wrapped phase and it is not necessary to locate branch cuts [6-8]. However, it is impossible to have a perfect phase-unwrapping system and there is a point where the noise makes this dynamic system start failing. Now we are going to show a strategy to make this dynamic system more robust to noise.

\section{B. Windowed Phase Unwrapping Following Iso-Phase Contours}

The windowed phase unwrapping uses the $2 \mathrm{D}$ firstorder dynamic system shown in Eq. (1). However, here we use it to unwrap the phase in a local window around a given site $(x, y)$. This process involves the following two steps:

1. Define $\Gamma[(x, y)]$ as the local window of dimension $N \times N$ around the site $(x, y)$.

2. Unwrap row by row all sites $\left(x^{\prime}, y^{\prime}\right) \in \Gamma[(x, y)]$ using the unwrapping system of Eq. (1).

These two basic steps define the windowed phaseunwrapping system; its output is a local unwrapped window $\Gamma[(x, y)]$ centered at position $(x, y)$. To cover all sites of the wrapped phase, it is necessary to move the unwrapping window scanning the sites of the wrapped phase. Note that we do not partition the wrapped phase in subwindows. Once we have unwrapped the phase in the local window $\Gamma[(x, y)]$, we move the window to the next site $\left(x^{\prime}, y^{\prime}\right)$ close to $(x, y)$ and apply the windowed phase unwrapping in $\Gamma\left[\left(x^{\prime}, y^{\prime}\right)\right]$. Consequently, if $\left(x^{\prime}, y^{\prime}\right) \in \Gamma[(x, y)]$, then $(x, y) \in \Gamma\left[\left(x^{\prime}, y^{\prime}\right)\right]$. Hence, a site $(x, y)$ is unwrapped and corrected several times as the window passes over it-since system (1) takes into account the status of its current neighborhood. This redundancy increases the stability of the dynamic phaseunwrapping system.

However, when the wrapped phase contains certain levels of noise, the success of the unwrapping depends on the path followed. Here we propose scanning the wrapped phase following its iso-phase contours. Using this strategy, the windowed phase unwrapping is enhanced because the phase on a contour remains constant and the variations between contours are smooth. As actually the wrapped phase traces the contours of the phase, we use the wrapped phase as the map to follow the contours. But, the noise in the wrapped phase may guide us off from a contour; then, we first filter the wrapped phase in the following way:

$$
p(x, y)=\phi(x, y) * h(x, y),
$$

where $*$ denotes convolution and $h(x, y)$ is the impulse response of a low-pass filter. In our case we use a Gaussian filter with parameter $\sigma$ for controlling its bandwidth. Note that $p(x, y)$ will be used just for scanning purposes; it is not used for unwrapping the phase. Thus, the windowed phase-unwrapping system following the iso-phase contours is as follows: 
1. Define $L$ as a list of $2 \mathrm{D}$ points and define visited $[(x, y)]$ as a flag function that is 1 if the point $(x, y)$ has been inserted to the list, and 0 otherwise.

2 . Set visited $[(x, y)]=0$ for all sites $(x, y)$ in the wrapped phase.

3. Take $P(x, y)=\|\nabla p(x, y)\|$; use finite differences for estimating the gradient $\nabla p(x, y)$.

4. Let $\left(x_{0}, y_{0}\right)$ be the starting point.

5. Pass the point $\left(x_{0}, y_{0}\right)$ to the windowed unwrapping system and set visited $[(x, y)]=1$.

6. From all points $(x, y)$ such that $\|(x, y)$ $-\left(x_{0}, y_{0}\right) \| \leq \sqrt{2}$, insert in the back of $L$ the point whose magnitude $P(x, y)$ is greater. The point $(x, y)$ is inserted to $L$ if and only if visited $[(x, y)] \neq 1$.

7. Set visited $[(x, y)]=1,(x, y)$ being the last point inserted in $L$.

8. Define $\left(x_{0}, y_{0}\right)=(m, n),(m, n)$ being the back point of the list $L$, and remove $(m, n)$ from the list.

9. Repeat steps 5 to 8 while $L$ is not empty.

This windowed phase-unwrapping system following iso-phase contours takes advantages of the following:

- The redundancy of the windowed phase unwrapping system corrects and unwraps a site several times as the window passes over it, making the system of Eq. (1) more robust.

- Following the iso-phase contours enhances the windowed phase unwrapping since the phase on a contour stems constant and has smooth variations between contours. The noise is filtered by the windowed phase-unwrapping system.

\section{Tests and Results}

The first test shows the performance of the windowed unwrapping system described here. In this test we want to show that the scanning strategy follows the iso-phase contours. We are going to unwrap a simulated wrapped phase using a $2 \mathrm{D}$ parabola as a model. In Fig. 1(a), we see the simulated wrapped parabola, and in Figs. 1(b) $-1(\mathrm{~h})$ we show snapshots of the scanning sequence. There we can see that the scanning strategy follows the iso-phase contours as expected. The parameters used in this test are $\tau=0.2, N=13, \sigma=21, \tau$ being the parameter of the dynamic system shown in Eq. (1), $N$ being the dimension of the square window used by the windowed unwrapping, and $\sigma$ being the parameter of the Gaussian filter used in Eq. (2). The starting point was the pixel located at site $(50,50)$ and the dimensions of the wrapped phase are $256 \times 256$ pixels.

The following tests show a comparison between the windowed phase-unwrapping method presented here and the phase-unwrapping method shown in [17]. Needless to say, as the noise level of these tests is high, the basic dynamic phase-unwrapping method presented in [12-14] does not work with these phase maps. On the other hand, the method presented in [17] shows a robust strategy to locate branch cuts and we compare with it because it tolerates high levels of noise. For these tests, we will use the wrapped phase maps shown in Fig. 2. These wrapped phase maps represent two different vibration modes taken by means of holography techniques using a postmortem chinchilla tympanic membrane as testing object. The phase maps were obtained using a stroboscopic double-exposure method $[18,19]$. The parameters used in the following tests are $\tau=0.07, N=13$, and $\sigma=41$ and starting point at pixel $(338,400)$. The dimensions of these phase maps are $800 \times 800$ pixels. In the first test we use the phase of Fig. 2(a). Figure 3(a) shows the unwrapped phase obtained with the method of [17] and Fig. 3(b) shows the unwrapped phase obtained with the herein-proposed method. Figures 3(c) and $3(d)$ show the rewrapped phase for comparison purposes. In Figs. 3(b) and 3(d) we can see clearly that the herein-proposed phase-unwrapping approach removes noise and unwraps the phase as expected. On the other hand, the method of [17] recovers the unwrapped phase with speckles. Actually, the speckles in the unwrapped phase of Fig. 3(a) are outliers that

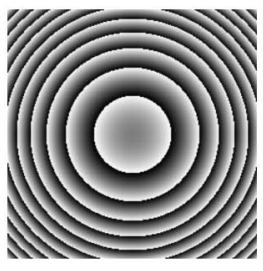

(a)

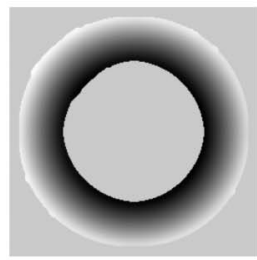

(e)

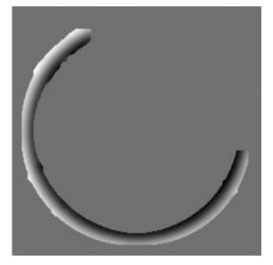

(b)

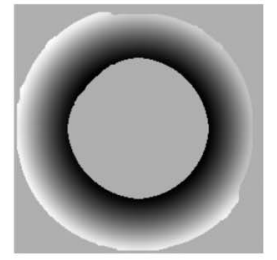

(f)

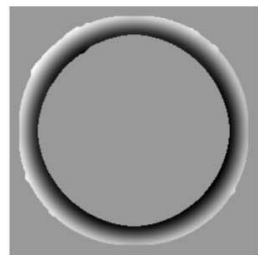

(c)

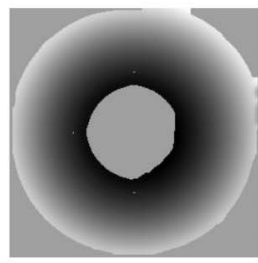

(g)

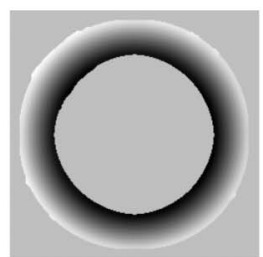

(d)

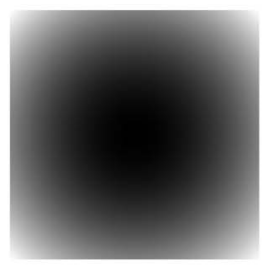

(h)

Fig. 1. Scanning the phase following its iso-phase contours. In (a) is the wrapped phase and in (b)-(h) are the snapshots of the scanning sequence applying the windowed phase-unwrapping algorithm. In (h) we see the global unwrapped phase as expected. 


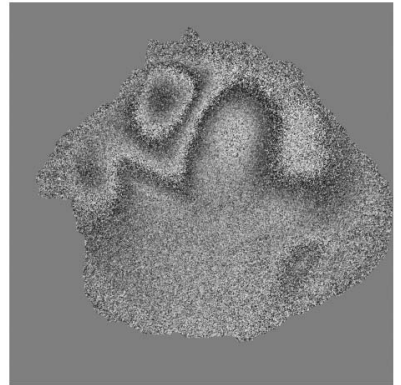

(a)

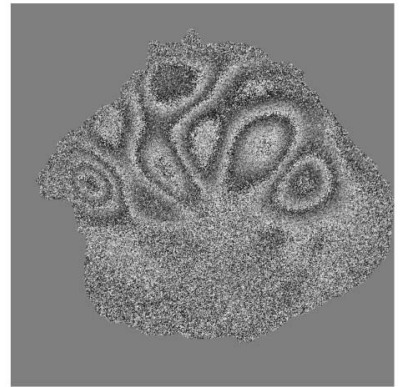

(b)

Fig. 2. Two experimentally obtained wrapped phase maps. These are vibration modes obtained from a cadaveric chinchilla tympanic membrane using a digital holography technique. The phase maps were obtained using a stroboscopic double-exposure method.

the unwrapping method was not able to unwrap. Using the windowed phase-unwrapping method described here, in Fig. 3(d) we can see that the dynamic range of the unwrapped phase is recovered correctly since it generates the same wrapped phase used as input, but without the noise. Now, the following test uses the wrapped phase of Fig. 2(b). In Fig. 4(a) we show the unwrapped phase obtained with the method of [17] and in Fig. 4(b) we show the unwrapped phase obtained with the method proposed here. In Figs. $4(\mathrm{c})$ and $\underline{4(\mathrm{~d})}$ we show the rewrapped phase for comparison purposes. Besides the speckles

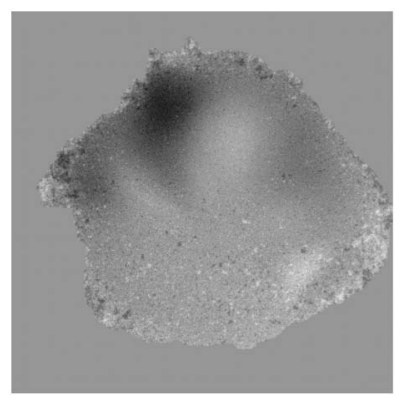

(a)

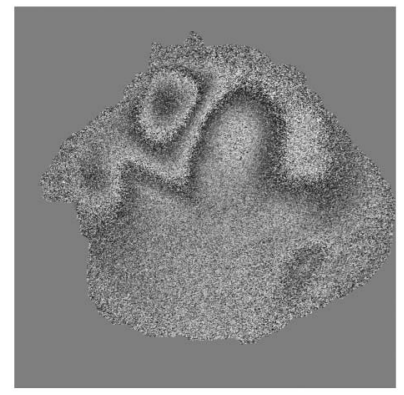

(c)

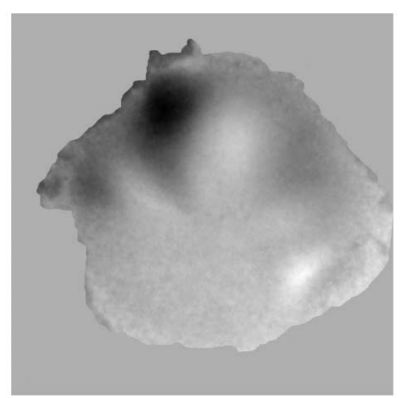

(b)

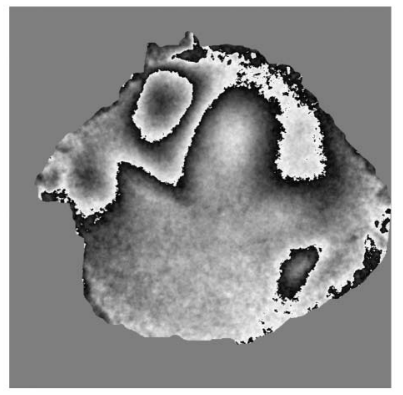

(d)

Fig. 3. Unwrapped phase from the phase map of Fig. 2(a). Panel (a) shows the unwrapped phase obtained with the branch-cut method of [17], panel (b) shows the unwrapped phase obtained with the windowed phase-unwrapped method proposed here. For illustration purposes, panels (c) and (d) show the unwrapped phase rewrapped.

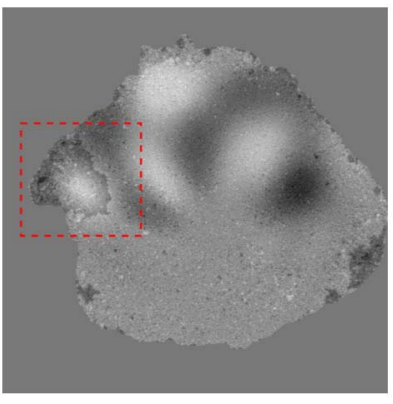

(a)

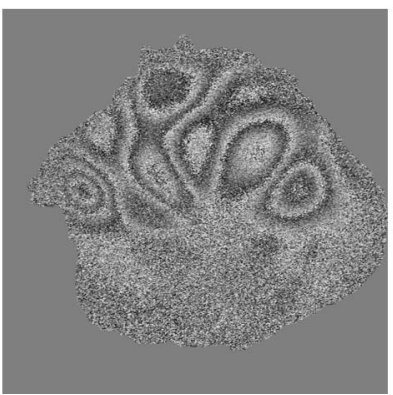

(c)

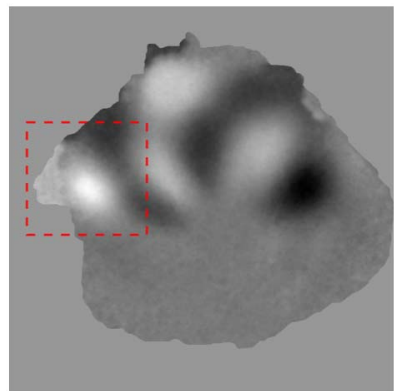

(b)

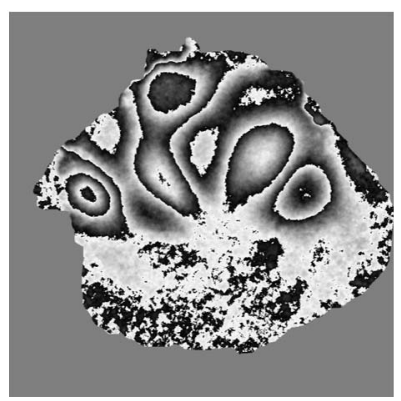

(d)
Fig. 4. (Color online) Unwrapped phase from the phase map of Fig. 2(a). Panel (a) shows the unwrapped phase obtained with the branch-cut method of [17], panel (b) shows the unwrapped phase obtained with the windowed phase unwrapped method proposed here. For illustration purposes, panels (c) and (d) show the unwrapped phase rewrapped.

obtained in the unwrapped phase of Fig. 4(a), we can see that the method of [17] recovers a mistaken unwrapped phase in the zone enclosed by the square of dashed lines. On the other hand, the hereinproposed windowed phase-unwrapping method obtains the unwrapped phase as expected.

\section{Discussion and Conclusions}

Let us discuss following iso-phase contours for processing the phase. Actually, this is not the first time that following iso-phase contours for processing the phase has been used as a scanning strategy. Most of the demodulation methods for single fringe patterns without carrier use a scanning strategy like this one [20-22]. This is because, under ideal conditions, the phase on an iso-phase contour does not change and makes more robust the processing. Following the iso-phase contours and using the windowed phase-unwrapping system, we obtain an enhanced and robust to noise dynamic phaseunwrapping system. Furthermore, the transitory response of the dynamic system of Eq. (1) is reduced since a pixel is processed several times as the window passes over it. The transitory response is a delay that generates the infinite impulse response systems when they start relaxed as in our case $[12,14]$.

As we said before, when the noise becomes a problem for the phase-unwrapping system, the success of the phase unwrapping depends on the path followed for scanning the phase. As the path to follow depends on the starting point, it may affect the phase 
unwrapping as well. We suggest to start in a point where the frequency of the phase is low. Finally, when the noise allows, we can use the windowed phase-unwrapping system scanning with a simple row-by-row strategy, improving in this way the results of the basic phase-unwrapping system [12-14]. But scanning following the fringes, we enhance the tolerance to noise of the phase-unwrapping system.

Summing up, we have presented a windowed phase-unwrapping system following the iso-phase contours of the wrapped phase. We compared this phase-unwrapping method with the method published in [17]. The wrapped phase maps used in the tests shown here have a level of noise that really represents a challenge for any robust phaseunwrapping system. The tests shown here demonstrate that this phase-unwrapping strategy is very robust to noise, filters noise, and obtains the unwrapped phase as expected.

\section{References}

1. D. Malacara, M. Servín, and Z. Malacara, Interferogram Analysis for Optical Testing (Taylor \& Francis, 2005).

2. M. Takeda, H. Ina, and S. Kobayashi, "Fourier-transform method of fringe-pattern analysis for computer-based topography and interferometry," J. Opt. Soc. Am. 72, 156-160 (1982).

3. M. Servin, J. C. Estrada, and J. A. Quiroga, "The general theory of phase shifting algorithms," Opt. Express 17, 21867-21881 (2009).

4. K. Itoh, "Analysis of the phase unwrapping algorithm," Appl. Opt. 21, 2470 (1982).

5. W. W. Macy, "Two-dimensional fringe-pattern analysis," Appl. Opt. 22, 3898-3901 (1983).

6. R. M. Goldstein, H. A. Zebker, and C. L. Werner, "Satellite radar interferometry: two-dimensional phase unwrapping," Radio Sci. 23, 713-720 (1988).

7. T. J. Flynn, "Two-dimensional phase unwrapping with minimum weighted discontinuity," J. Opt. Soc. Am. A 14, 2692-2701 (1997).
8. D. C. Ghiglia and M. D. Pritt, Two-Dimensional Phase Unwrapping; Theory, Algorithms, and Software (WileyInterscience, 1998).

9. D. C. Ghiglia and L. A. Romero, "Robust two-dimensional weighted and unweighted phase unwrapping that uses fast transforms and iterative methods," J. Opt. Soc. Am. A 11, 107-117 (1994).

10. J. L. Marroquin, M. Tapia, R. Rodriguez-Vera, and M. Servin, "Parallel algorithms for phase unwrapping based on Markov random field models," J. Opt. Soc. Am. A 12, 2578-2585 (1995).

11. J. L. Marroquin and M. Rivera, "Quadratic regularization functionals for phase unwrapping," J. Opt. Soc. Am. A 12 , 2393-2400 (1995).

12. J. C. Estrada, M. Servin, and J. A. Quiroga, "Noise robust linear dynamic system for phase unwrapping and smoothing," Opt. Express 19, 5126-5133 (2011).

13. M. A. Navarro, J. C. Estrada, M. Servin, J. A. Quiroga, and J. Vargas, "Fast two-dimensional simultaneous phase unwrapping and low-pass filtering," Opt. Express 20, 2556-2561 (2012).

14. J. C. Estrada, M. Servin, and J. Vargas, "2D simultaneous phase unwrapping and filtering: A review and comparison," Opt. Lasers Eng. 50, 1026-1029 (2012).

15. B. Strobel, "Processing of interferometric phase maps as complex-valued phasor images," Appl. Opt. 35, 2192-2198 (1996).

16. J. C. Estrada, "Phase unwrapping software," http://goo.gl/ RW2nQ.

17. J. R. Buckland, J. M. Huntley, and S. R. E. Turner, "Unwrapping noisy phase maps by use of a minimum-cost-matching algorithm," Appl. Opt. 34, 5100-5108 (1995).

18. K. A. Stenson and W. R. Brohinsky, "Electro-optic holography system for vibration analysis and nondestructive testing," Opt. Eng. 26, 1234-1239 (1987).

19. P. Hariharan, B. F. Oreb, and C. H. Freund, "Stroboscopic holographic interferometry: measurements of vector components of vibration," Appl. Opt. 26, 3899-3903 (1987).

20. M. Servin, J. L. Marroquin, D. Malacara, and F. J. Cuevas, "Phase unwrapping with a regularized phase-tracking system," Appl. Opt. 37, 1917-1923 (1998).

21. M. Servin, F. J. Cuevas, D. Malacara, J. L. Marroquin, and R. Rodriguez-Vera, "Phase unwrapping through demodulation by use of the regularized phase-tracking technique," Appl. Opt. 38, 1934-1941 (1999).

22. J. C. Estrada, M. Servin, J. L. Marroquin, and J. L. Marroquín, "Local adaptable quadrature filters to demodulate single fringe patterns with closed fringes," Opt. Express 15, 2288-2298 (2007). 